\title{
Atividade Física e Câncer Colorretal: Estudo de Caso-Controle no Município de Pelotas
}

doi: https://doi.org/10.32635/2176-9745.RBC.2021v67n4.1457

\author{
Physical Activity and Colorretal Cancer: Case-Control Study in the City of Pelotas \\ Actividad Física y Cáncer de Colorretal: Estudio de Caso-Control en la Ciudad de Pelotas
}

Daniele Barin Facin'; Maria Laura Brizio Gomes²; Marlos Rodrigues Domingues ${ }^{3}$

\section{RESUMO}

Introduçáo: Neoplasias são um dos principais problemas de saúde prevalentes. O câncer colorretal (CCR) apresenta alta incidência no Brasil, o tratamento da doença consiste em auxiliar o sistema imunológico a combater as células malignas, e a prática de exercícios pode auxiliar em sua prevenção. $\mathrm{Na}$ busca de fatores de risco para CCR, pesquisas obtiveram dados consistentes quanto a: alimentação inapropriada, inatividade física, obesidade, consumo de álcool e tabaco, mas ainda não foi possível encontrar dados sobre o histórico de atividade física (AF) dos pacientes diagnosticados com CCR. Objetivo: Avaliar se a prática de AF durante toda a vida pode influenciar no desenvolvimento da doença. Método: Estudo caso-controle. Os casos foram obtidos nos centros oncológicos de Pelotas, Brasil. Dois controles de cada caso foram detectados no mesmo centro de saúde. Ambos os grupos foram pareados por idade $( \pm 5$ anos). Ao todo, participaram do estudo 82 casos e 165 controles. Resultados: A partir das análises brutas e ajustadas para os fatores de confusão, a AF em seus quatro domínios e a AF total, realizadas durante toda a vida, não estiveram associadas ao desfecho. Conclusáo: Os resultados analisados neste estudo foram contrários, em sua maior parte, ao encontrado na literatura; as possíveis explicaçóes foram o perfil de AF na população estudada, as características da AF ocupacional e o tamanho amostral.

Palavras-chave: Neoplasias Colorretais; Exercício Físico; Fatores de Risco; Estudos de Casos e Controles.

\section{ABSTRACT}

Introduction: Neoplasms are one of the main prevalent health problems. Colorectal cancer (CRC) has a high incidence in Brazil, the treatment of the disease consists in helping the immune system to fight the malignant cells, and the practice of exercises can help its prevention. Studies addressing the risk factors for CRC found consistent data about inadequate diet, physical inactivity, obesity, alcohol, and tobacco use, but it was not possible to find data on the history of physical activity (PA) of patients diagnosed with CRC in Brazil. Objective: The aim of this study was to evaluate whether physical activity (PA) during the lifespan can influence the likelihood of developing the disease. Method: A case-control study was carried out. Cases were obtained from oncologic health centers in Pelotas, Brazil. Two controls were found for each case in the same health center. Both groups were age-matched ( \pm 5 years), 82 cases and 165 controls participated in the study. Results: Based in the raw and adjusted analyzes for the confounding factors, PA in its four domains and total PA performed throughout life were not associated with the outcome. Conclusion: The results analyzed in this study were, mostly, contrary to those found in the literature, with possible explanations being the profile of PA of the population studied, the characteristics of occupational physical activity and the sample size. Key words: Colorectal Neoplasms; Exercise; Risk Factors; Case-Control Studies.

\section{RESUMEN}

Introducción: Neoplasias son uno de los principales problemas de salud prevalentes. Cáncer colorrectal (CCR) tiene una alta incidencia en Brasil, el tratamiento consiste en ayudar al sistema inmunológico en la defensa de las células malignas y la práctica de ejercicios puede ayudar en su prevención. Búsqueda de factores de riesgo para CCR, investigación ha obtenido datos consistentes sobre dieta inadecuada, inactividad física, obesidad, consumo de alcohol y tabaco, pero aún no ha sido posible encontrar datos brasileños sobre la historia de actividad física (AF) de los pacientes diagnosticado con CCR. Objetivo: El estudio tuvo como objetivo evaluar si la práctica de AF a lo largo de la vida puede influir en la probabilidad de desarrollar la enfermedad. Método: Realizó un estudio de casos y controles. Los casos fueron reclutados en centros de oncología en Pelotas, Brasil. Cada caso tuvo dos controles, que se encuentran en el mismo centro de salud. Ambos grupos fueron emparejados por edad ( \pm-5 años), 82 casos y 165 controles participaron en el estudio. Resultados: Análisis crudos y ajustados para los factores de confusión, AF en sus cuatro dominios y AF total realizada durante toda la vida no se asociaron con el resultado. Conclusión: Resultados analizados en este estudio fueron contrarios, en su mayor parte, a los encontrados en la literatura, siendo posibles explicaciones el perfil de actividad física en la población estudiada, las características de la AF ocupacional y el tamaño de la muestra.

Palabras clave: Neoplasias Colorrectales; Ejercicio Físico; Factores de Riesgo; Estudios de Casos y Controles.

\footnotetext{
1-3Universidade Federal de Pelotas. Programa de Pós-graduação em Educação Física. E-mails: danibarin@yahoo.com.br; marialresem@hotmail.com; marlosufpel@gmail.com. Orcid iD: https://orcid.org/0000-0002-5879-147X; Orcid iD: https://orcid.org/0000-0002-5110-5529; Orcid iD: https://orcid.org/0000-0002-2503-2944 Endereço para correspondência: Daniele Barin Facin. Rua Machado de Assis, 364 - apto. 102 - Menino Jesus. Santa Maria (RS), Brasil. CEP 97050-450. E-mail: danibarin@yahoo.com.br
} 


\section{INTRODUÇÃO}

No Brasil, o progressivo processo de industrializaçáo e a globalização mudaram os paradigmas de mortalidade. No início do século XX, se evidenciavam as doenças infectocontagiosas entre os principais problemas de saúde pública, responsáveis por elevadas taxas de mortalidade infantil e baixa expectativa de vida da população ${ }^{1}$. Atualmente, o câncer é o principal problema de saúde pública no mundo, em razão do envelhecimento, do crescimento populacional, da mudança na distribuição e na prevalência dos fatores de risco de câncer, especialmente aos associados ao desenvolvimento socioeconômico. Verifica-se também um declínio de câncer associado a infecçóes e um aumento daqueles associados à melhoria das condiçôes socioeconômicas, com a incorporação de hábitos e atitudes associados à urbanização (sedentarismo, alimentação inadequada, entre outros) ${ }^{2}$.

Entre as principais neoplasias incidentes, o câncer colorretal (CCR) se caracteriza pelo desenvolvimento de tumores malignos no cólon e/ou reto. Seus sintomas são pouco perceptíveis e, quando se tornam mais evidentes, a neoplasia já está no grau mais avançado. Dados epidemiológicos da doença mostram alta incidência no Brasil, onde, segundo informaçóes de base populacional, em 2015, ocorreram 151/100 mil habitantes novos casos. $\mathrm{Na}$ incidência mundial, de acordo com o GLOBOCAN 2008, estáo os tumores de colón e reto em terceiro lugar, com 1,2 milhão de casos novos e 680 mil óbitos $^{3}$. Com destaque para as estimativas de 2020 no Estado do Rio Grande do Sul, onde o CCR apresentou altas taxas brutas de incidência (em homens, 1.300/100 mil habitantes; em mulheres, 1.240/100 mil habitantes), sendo o quarto tipo de câncer mais frequente nessa Região, em ambos os sexos ${ }^{2}$.

Contudo, quando o assunto são neoplasias, ainda há dificuldade para encontrar soluçóes para preveni-las, sendo frequentes as mortes por sua causa em muitos países. O tratamento da doença consiste em auxiliar o sistema imune na defesa das células malignas, e a prática de exercícios também pode auxiliar nesse processo ${ }^{4}$. No que diz respeito ao CCR, a literatura afirma que se trata de uma doença multifatorial influenciada por fatores genéticos, ambientais e relacionados ao estilo de vida ${ }^{5,6}$.

A AF regular pode prevenir e ajudar a controlar doenças cardíacas, diabetes tipo 2 e câncer (bexiga, mama, cólon, endométrio, adenocarcinoma, esofágico, estômago e rim), que causam quase três quartos das mortes em todo o mundo 7 .

Segundo as recomendaçóes mundiais, adultos devem realizar semanalmente pelo menos 150 a 300 minutos de AF aeróbica de moderada intensidade ou pelo menos
75 a 150 minutos de vigorosa intensidade 7 , valores estes que não são atingidos na maioria das capitais brasileiras ${ }^{8}$. Em países desenvolvidos, a alta incidência do CCR está associada ao estilo de vida e a fatores como dieta hipercalórica e rica em carne vermelha. Por outro lado, um peso corporal dentro de padróes normais (índice de massa corporal - IMC 20-25), AF, e uma dieta saudável podem diminuir o risco de CCR em $60 \%$ a $80 \%$.

Diversos estudos demonstraram uma relação de proteção entre a prática de $\mathrm{AF}$ e o desenvolvimento de $\mathrm{CCR}^{10-17}$. Porém, em países em desenvolvimento, esse tema foi pouco abordado, e o estilo de vida dessas populaçôes difere em diversos fatores. Considerando os potenciais impactos da referida doença e seus dados epidemiológicos, é essencial que os profissionais da saúde tenham orientaçóes quanto a possíveis variáveis que possam auxiliar a prevenir a referida doença. Assim, o objetivo deste estudo foi verificar a associação entre a prática de AF durante toda vida e a incidência de CCR no Sul do Brasil.

\section{MÉTODO}

Este estudo utilizou o delineamento caso-controle, considerando como casos os pacientes com CCR e como controles, os indivíduos que estavam sendo tratados no mesmo centro de saúde que os casos, porém acometidos por outras enfermidades, como também os acompanhantes dos pacientes. Não houve restrição de sexo na amostra e indivíduos com mais de 18 anos foram selecionados. O delineamento utilizado permitiu uma abordagem retrospectiva da amostra sobre as variáveis que foram analisadas. A principal variável de exposição estudada foi a AF desenvolvida ao longo da vida nos domínios: lazer, ocupacional, doméstico e no deslocamento. A pesquisa propôs a análise da associação entre a prática da $\mathrm{AF}$ e a ocorrência do CCR. Os dados dos casos foram obtidos por meio dos centros de saúde oncológicos, públicos e privados, da cidade de Pelotas. As entrevistas foram realizadas mediante seleção dos casos nos prontuários fornecidos pelas instituiçóes. Os questionários foram aplicados nos casos durante a sessão de quimioterapia ou durante a internaçáo hospitalar e, a partir de cada caso, foram selecionados dois controles, pareados por sexo e por idade ( \pm 5 anos), estes obtidos nos mesmos centros de saúde dos casos. Somente indivíduos acometidos por CCR primário foram incluídos na amostra, entre eles, casos incidentes e prevalentes. Indivíduos que apresentaram câncer de foco secundário foram excluídos.

Os casos e controles responderam ao questionário contendo questôes sobre características sociodemográficas, sexo, idade e cor da pele, variáveis antropométricas 
(altura e peso), situação conjugal, escolaridade, características comportamentais (dieta, tabagismo, consumo de álcool e AF) e histórico familiar de câncer. A variável "atividade física por toda vida" foi avaliada por meio do questionário proposto por Friedenreich et al. ${ }^{18}$ (Division of Epidemiology, Prevention and Screening, Alberta Cancer Board, Canada), chamado - "The lifetime total physical activity questionnaire" (questionário de atividade física durante toda vida), que englobou os quatro domínios da AF, levando em conta atividades domésticas, ocupacionais, de lazer e de deslocamento realizadas durante toda vida. Além disso, foram mensuradas a frequência (vezes na semana), a duração (tempo) e a intensidade (em múltiplos equivalentes metabólicos - MET) de cada atividade, fornecendo um escore de atividade para toda a vida.

$\mathrm{O}$ instrumento forneceu a quantificação da $\mathrm{AF}$ em forma de MET. Um MET equivale ao número de calorias que um corpo consome enquanto está em repouso ${ }^{19}$. Os MET são incrementados na medida em que aumenta a intensidade da atividade. A criação do escore de AF leva em conta o número de meses/anos em que a pessoa realizou tal atividade, contabilizando os valores usuais de frequência semanal, duração e o tipo da atividade, possibilitando associar a atividade a um gasto em MET.

A escolha das variáveis foi realizada de acordo com uma revisão de literatura sobre os principais fatores de risco (características sociodemográficas, dieta, obesidade, $\mathrm{AF}$ e comportamento sedentário, álcool e tabaco) para CCR. Náo foram coletados dados de exames preventivos, como colonoscopia, pois foi analisado o histórico pregresso da amostra.

As características sociodemográficas foram analisadas, o nível socioeconômico determinado pelo índice de bens dos participantes (análise de componentes principais de um inventário de utensílios domésticos) e a cor da pele definida a partir da observação do entrevistador. Foram coletados dados sobre escolaridade, variáveis antropométricas (altura e peso), situação conjugal, características comportamentais, histórico familiar de câncer, sexo e idade. As variáveis comportamentais e antropométricas foram coletadas por intermédio do questionário e do autorrelato de peso e altura, sendo que, nos casos, foi coletada a média de peso antes e após a doença. A dieta foi composta pela análise do consumo da frequência mensal e de toda a vida dos alimentos: carne de boi, frango, porco, peixe, frituras, legumes, verduras, frutas, doces, sucos de frutas industrializados e refrigerante.

A coleta dos dados ocorreu durante oito meses, no período de outubro de 2018 a maio de 2019, totalizando 82 casos e 165 controles obtidos dos serviços de saúde.
Os dados foram digitados em uma planilha Excel (Microsoft Excel 2010), e a análise estatística foi realizada com o pacote estatístico Stata 15.0 for Windows (Stata Corporation). Foram construídos escores de AF em MET e análises bivariadas por regressão logística, seguidas por uma análise multivariável por regressão logística condicional (pareamento para sexo e idade), que serviu para controlar possíveis fatores de confusão.

As variáveis foram testadas uma a uma para medir sua associação com o desfecho. Variáveis com valor de $\mathrm{p}$ inferior a 0,20 foram levadas para o modelo de regressão e foram excluídas quando o valor de $\mathrm{p}$ foi superior a 0,20 depois de ajuste. Após a composição do modelo de regressão final, as variáveis de AF foram testadas uma a uma (independente do seu valor na análise bruta) para medir a associação dos domínios da AF ajustados para os potenciais fatores de confusão.

Por intermédio de um cálculo de poder estatístico feito posteriormente às análises, observou-se que o tamanho amostral obtido, aliado às características de $\mathrm{AF}$ dos sujeitos, conferiu um poder estatístico abaixo de 50\%.

Este estudo foi aprovado pelo Comitê de Ética em Pesquisa da Escola Superior de Educação Física da Universidade Federal de Pelotas (CAEE 94588318.6.0000.5313).

Foi solicitado aos entrevistados seu consentimento verbal para a realização da coleta das informaçóes, seguido da assinatura de um Termo de Consentimento, Livre e Esclarecido, confirmando sua escolha voluntária em participar do estudo, de forma que qualquer indivíduo poderia recusar-se a participar da pesquisa.

\section{RESULTADOS}

As características pessoais, sociodemográficas, comportamentais e antropométricas da amostra foram examinadas para comparar casos e controles. Participantes de estudos de casos e controles, em sua grande maioria, eram da cor branca, casados e com idade média de aproximadamente 59 anos $( \pm 12,95)$. Náo houve diferença significativa entre casos e controles referente ao sexo, idade, cor da pele, estado civil, escolaridade, nível socioeconômico (índice de bens), consumo de álcool, tabagismo, IMC. Como características da amostra, foi observado que os casos, em sua maior parte, eram exfumantes e apresentaram maior percentual de sobrepeso (segundo o cálculo do IMC) (Tabela 1).

$\mathrm{Na}$ análise bruta dos fatores de risco relacionados aos aspectos sociodemográficos, comportamentais e antropométricos para o CCR, as variáveis sociodemográficas, comportamentais e o IMC não foram associados ao desfecho, porém as variáveis da dieta: 
Facin DB, Gomes MLB, Domingues MR

Tabela 1. Descrição das características sociodemográficas e comportamentais da amostra. Pelotas, 2019 ( $n=247)$

\begin{tabular}{|c|c|c|c|}
\hline Características & Amostra total (\%) & Casos (\%) & Valor $p$ \\
\hline Sexo & & & $0,96^{2}$ \\
\hline Masculino & 50,2 & 33,1 & \\
\hline Feminino & 49,8 & 33,3 & \\
\hline Idade (anos) & & & $0,47^{1}$ \\
\hline $19-50$ & 23,5 & 31,0 & \\
\hline $51-65$ & 40,9 & 31,7 & \\
\hline $66-88$ & 35,6 & 36,4 & \\
\hline Cor da pele & & & $0,70^{2}$ \\
\hline Branca & 63,5 & 34,4 & \\
\hline Preta & 13,8 & 35,3 & \\
\hline Parda/morena & 22,7 & 28,6 & \\
\hline Estado civil & & & $0,41^{2}$ \\
\hline Casado & 68,0 & 30,4 & \\
\hline Solteiro & 8,9 & 31,8 & \\
\hline Divorciado & 11,3 & 39,3 & \\
\hline Viúvo & 11,8 & 44,8 & \\
\hline Escolaridade (anos) & & & 0,991 \\
\hline Até 5 & 55,1 & 33,1 & \\
\hline $6-10$ & 17,0 & 38,1 & \\
\hline $11-15$ & 25,1 & 27,4 & \\
\hline $16-19$ & 2,8 & 57,1 & \\
\hline Índice de bens (quartis) & & & $0,08^{1}$ \\
\hline $1^{\circ}$ (mais pobres) & 25,1 & 24,2 & \\
\hline $2^{\circ}$ & 25,1 & 30,7 & \\
\hline $3^{\circ}$ & 25,1 & 41,9 & \\
\hline $4^{\circ}$ (mais ricos) & 24,7 & 36,1 & \\
\hline Histórico de tabagismo & & & $0,15^{2}$ \\
\hline Fumantes & 8,1 & 20,0 & \\
\hline Nunca fumantes & 42,5 & 29,5 & \\
\hline Ex-fumantes & 49,4 & 38,5 & \\
\hline Consumo de álcool & & & $0,42^{2}$ \\
\hline Sim & 87,9 & 26,7 & \\
\hline Não & 12,1 & 34,1 & \\
\hline Índice de massa corporal $\left(\mathbf{k g} / \mathbf{m}^{2}\right)$ & & & $0,36^{1}$ \\
\hline Peso normal & 43,3 & 29,9 & \\
\hline Sobrepeso & 35,6 & 35,2 & \\
\hline Obesidade grau 1 & 21,1 & 36,5 & \\
\hline
\end{tabular}

(1) Teste qui-quadrado de tendência linear.

(2) Teste qui-quadrado de heterogeneidade.

consumo de refrigerante toda vida $(\mathrm{p}=0,04)$ e consumo de peixe toda vida $(\mathrm{p}=0,001)$ foram associadas ao desfecho, no sentido de aumentar o risco de câncer.

Em relaçáo ao perfil de AF da amostra, no domínio lazer, $25 \%$ dos sujeitos foram inativos ao longo de toda vida e $85,8 \%$ relataram $\mathrm{AF}$ domésticas; na $\mathrm{AF}$ de deslocamento, 44,5\% realizavam transporte ativo e, no domínio ocupacional, 95,9\% foram ativos fisicamente, sendo que $32 \%$ da amostra relataram trabalho na lavoura extenuante e com exposição a pesticidas. A Tabela 2 apresenta os dados da análise bruta e ajustada da AF. Em ambas as análises, a AF nos quatro domínios (lazer, 
doméstico, deslocamento e ocupacional) e a $\mathrm{AF}$ total não se mostraram associadas ao desfecho.

\section{DISCUSSÃO}

As variáveis sociodemográficas e comportamentais do estudo não se mostraram associadas ao desfecho. Ao contrário do encontrado na literatura, a respeito do efeito do tabagismo, consumo de álcool e de carne vermelha no desenvolvimento de CCR, que apresentaram diferença significativa em diversos estudos ${ }^{20-23}$; já outro estudo do tipo caso-controle náo encontrou diferença significativa para o aumento do risco de CCR frente ao tabagismo, etilismo e consumo de fibras ${ }^{24}$, o que torna essas variáveis inconsistentes diante da doença analisada. Em relação ao IMC, o presente estudo não encontrou associação significativa, porém, em um estudo caso-controle, foi analisado o IMC em homens obesos, que se mostrou positivamente associado ao risco de desenvolver $\mathrm{CCR}^{25}$.

Em relação à dieta, este estudo apresentou em sua análise bruta uma diferença significativa no consumo por toda a vida de refrigerante $(\mathrm{p}=0,04)$ e peixe $(\mathrm{p}=0,001)$, obtendo maiores chances de desenvolver CCR. No que diz respeito ao consumo de peixe, diversos estudos foram contrários ao encontrado nesta pesquisa, obtendo-se resultados com redução significativa do risco de CCR na sua ingestão ${ }^{16,26}$; no entanto, especula-se que o modo de preparo do peixe (majoritariamente frito em imersão)

Tabela 2. Análise bruta e ajustada da ocorrência de câncer colorretal de acordo com os domínios da atividade física ao longo da vida. Odds ratios e intervalos de confiança de 95\% obtidos por regressão logística condicional, pareada por sexo e idade. Pelotas, 2019 (

\begin{tabular}{|c|c|c|c|c|}
\hline Atividade Física (quartis) & OR $_{\text {bruta }}$ (IC95\%) & Valor $\mathbf{p}$ & OR $_{\text {aiustada }}($ IC95\%) & Valor $p$ \\
\hline Lazer & & 0,53 & & 0,55 \\
\hline $1^{\circ}$ (menos ativos) & 1,0 & & 1,0 & \\
\hline $2^{\circ}$ & $1,49(0,67-3,30)$ & & $1,30(0,51-3,30)$ & \\
\hline $3^{\circ}$ & $1,09(0,49-2,42)$ & & $0,95(0,39-2,32)$ & \\
\hline $4^{\circ}$ (mais ativos) & $1,61(0,64-3,24)$ & & $1,56(0,58-4,20)$ & \\
\hline Doméstica & & 0,51 & & 0,64 \\
\hline $1^{\circ}$ (menos ativos) & 1,0 & & 1,0 & \\
\hline $2^{\circ}$ & $0,46(0,21-1,04)$ & & $0,55(0,22-1,33)$ & \\
\hline $3^{\circ}$ & $0,40(0,30-1,65)$ & & $0,48(0,18-1,26)$ & \\
\hline $4^{\circ}$ (mais ativos) & $0,71(0,30-1,65)$ & & $0,78(0,29-2,08)$ & \\
\hline Deslocamento & & 0,44 & & 0,38 \\
\hline $1^{\circ}$ (menos ativos) & 1,0 & & 1,00 & \\
\hline $2^{\circ}$ & $0,96(0,29-3,12)$ & & $1,16(0,32-4,22)$ & \\
\hline $3^{\circ}$ & $0,68(0,34-1,37)$ & & $0,46(0,20-1,06)$ & \\
\hline $4^{\circ}$ (mais ativos) & $0,86(0,43-1,73)$ & & $0,96(0,42-2,22)$ & \\
\hline Ocupacional & & 0,69 & & 0,61 \\
\hline $1^{\circ}$ (menos ativos) & 1,0 & & 1,0 & \\
\hline $2^{\circ}$ & $2,43(0,97-6,05)$ & & $2,56(0,90-7,27)$ & \\
\hline $3^{\circ}$ & $1,30(0,52-3,22)$ & & $1,30(0,46-3,68)$ & \\
\hline $4^{\circ}$ (mais ativos) & $1,83(0,71-4,73)$ & & $2,04(0,64-6,45)$ & \\
\hline Atividade física total & & 0,83 & & 0,91 \\
\hline $1^{\circ}$ (menos ativos) & 1,0 & & 1,0 & \\
\hline $2^{\circ}$ & $0,59(0,26-1,36)$ & & $0,69(0,27-1,77)$ & \\
\hline $3^{\circ}$ & $0,91(0,42-1,98)$ & & $0,90(0,35-2,30)$ & \\
\hline $4^{\circ}$ (mais ativos) & $0,76(0,33-1,73)$ & & $0,83(0,31-2,28)$ & \\
\hline
\end{tabular}

Legendas: OR = Odds ratios; IC95\% = Intervalo de confiança de 95\%.

$\left(^{*}\right)$ Variáveis de atividade física ajustadas para os seguintes fatores de confusão índice de bens, tabagismo, consumo de carne suína, doces, frutas, refrigerante e peixe ao longo da vida. 
possa ter alguma implicação nessa associação. Não foram encontrados estudos acerca do consumo de refrigerante, porém alguns autores encontraram associação em relação à alta ingestão de alimentos com alto índice glicêmico, o que poderia explicar parcialmente essa associação observada $^{27,28}$.

A principal variável de exposição mensurada no presente estudo foi a AF em seus quatro domínios e total; e, após análise ajustada para os fatores de confusão, esta não apresentou associação significativa com o desfecho do estudo. Apesar de as medidas de efeito indicarem odds ratios na proteção para os domínios de deslocamento, para atividades domésticas e atividade total na vida, não foi detectada associação significativa entre qualquer domínio de AF e desfecho CCR.

Algumas das especulaçóes sobre esses achados são embasadas nas características específicas da amostra, que apresentou um nível muito baixo de AF, sendo aparentemente uma população bastante inativa no lazer e com grande carga de atividades ocupacionais, o que pode influenciar bastante os desfechos em saúde.

Este estudo apresenta limitaçóes que são inerentes aos estudos de caso-controle, um delineamento que requer que os avaliados se recordem de eventos e comportamentos de um passado longínquo, representando viés recordatório, viés de informaçấo e consequente potencial viés de classificação. Apesar dessa limitação, tal delineamento é o mais indicado para avaliar esse tipo de associação, em virtude do longo período de induçáo e latência do desfecho.

Metanálises sobre AF e câncer encontraram resultados diferentes do presente estudo ${ }^{14,29,30}$. Liu et al. ${ }^{29}$ estimaram o risco de câncer e atividade física de lazer (AFL) em 126 estudos. As horas semanais de AFL relatadas foram multiplicadas por 8 MET para atividades vigorosas; 6 MET para atividades moderadas a vigorosas; e 4 MET para atividades moderadas, demonstrando que o risco total de câncer foi reduzido em $10 \%$ em pessoas que realizaram o maior tempo de AFL em comparação com aqueles que fizeram o mínimo ${ }^{29}$. Diferente análise conjunta de dados sobre a AFL com 12 estudos prospectivos dos EUA e estudos de coorte europeus obtiveram nível de AFL de $8 \mathrm{MET}$-h/semana, o equivalente a 150 minutos/semana de AF com intensidade moderada, associado a um menor risco para 13 tipos de cânceres, em especial o câncer de cólon, que apresentou $16 \%$ de proteção (hazard ratio $\mathrm{HR}=0,84 ; \mathrm{IC} 0,77-0,91$ ), quando comparados indivíduos mais ativos aos menos ativos ${ }^{31}$.

Uma pesquisa realizada com dados de uma coorte constatou que a AF acima de 21 MET-horas/semana esteve associada a uma reduçáo do risco de CCR em $39 \%(\mathrm{HR}=0,61 ; \mathrm{p}<0,0001)$ e câncer de cólon em 45\%
$(\mathrm{HR}=0,55 ; \mathrm{p}<0,0001)^{20}$. Uma coorte do Nurses' Health Study encontrou resultados protetivos para AFL. Na análise ajustada, ao se relacionar o nível de AF mais alto versus o mais baixo, obteve-se reduçáo do risco para CCR na AFL em 29\% (HR=0,7; IC95\% 0,49-1,28; $\mathrm{p}=0,03)$, porém os outros domínios da $\mathrm{AF}$ não apresentaram valor significativo ${ }^{32}$.

O presente trabalho se baseou em uma análise pareada por sexo. Alguns estudos já relataram resultados distintos entre homens e mulheres, por exemplo, Keum et al. ${ }^{33}$ analisaram a redução do risco de câncer frente à $\mathrm{AF}$ em uma coorte prospectiva que acompanhou 43.479 homens e examinaram a associação entre $\mathrm{AF}$ e risco de câncer no aparelho digestivo. Obtendo como resultados que níveis mais altos de $\mathrm{AF}$ foram associados a $26 \%(\mathrm{HR}=0,74$ para $\geq 63,0$ vs. $\leq 8,9$ MET-horas/semana; IC95\% 0,59-0,93) de proteção contra o câncer no sistema digestivo. Sendo que o exercício aeróbico (aproximadamente 30 METhoras/semana -1 MET, ou o equivalente metabólico, corresponde ao consumo de $3,5 \mathrm{~mL}$ de oxigênio para cada $\mathrm{kg}$ de massa corporal a cada minuto) obteve proteção de 32\% $(\mathrm{HR}=0,68$; IC95\% 0,56-0,83) contra o câncer do sistema digestivo ${ }^{33,34}$. Diferente estudo de coorte verificou que homens com baixa AF (8,3 MET-h/ semana) obtiveram 31\% (HR=0,69; IC95\% 1,00$1,70)$ no aumento do risco de CCR em comparação a participantes com AF elevada (>16,6 METh/semana), porém não encontraram associação com a incidência de câncer entre as mulheres ${ }^{35}$. Resultados semelhantes foram encontrados em um estudo de coorte prospectivo no qual não foram encontradas associaçôes entre o nível de $\mathrm{AF}$ e o risco de câncer de cólon, comparando mulheres com AF nível 1-2 àquelas com nível de AF 5-6 (linha de base: $\mathrm{HR}=0,90 ; \mathrm{IC} 95 \% 0,66-1,23 ; \mathrm{p}=0,76$; mediçóes repetidas: $\mathrm{HR}=0,78$; IC95\% 0,55-1,10; $\mathrm{p}=0,27)$. Os resultados foram os mesmos quando se comparou AF nível 9-10 para o nível de referência (linha de base: $\mathrm{HR}=0,80$; IC95\% 0,56-1,12; $\mathrm{p}=0,76$; medidas repetidas: $\mathrm{HR}=0,82$; IC95\% $0,58-1,16 ; \mathrm{p}=0,27)^{36}$. Porém, em diferente pesquisa, as mulheres que praticavam AF em um nível de 21 METhoras/semana tiveram uma redução de $49 \%$ no risco em comparação a mulheres que praticaram apenas 2 METhoras de atividade por semana ${ }^{22}$. Segundo essas pesquisas, pode-se dizer que a AF apresenta proteção frente ao CCR em homens, porém náo é possível essa afirmação no caso de mulheres, em razão de as pesquisas apresentarem resultados controversos.

Muitos mecanismos biológicos têm sido propostos para explicar a associaçáo entre a AF e o CCR. Um deles é a capacidade da inatividade física aumentar a prevalência de câncer de cólon, por meio de níveis mais elevados de insulina no sangue, o que, em longo prazo, pode resultar 
em resistência insulínica - um conhecido fator de risco para câncer. Além disso, pode causar níveis mais altos de IGF-1 (fator de crescimento semelhante à insulina tipo 1 na corrente sanguínea, expondo o rápido retorno no epitélio do cólon a níveis mais altos de hormônio anabólico associado à maior incidência de câncer de cólon $)^{11,12}$. Como também, a AF pode influenciar diretamente no risco de câncer, uma vez que diminui o tempo de trânsito intestinal e, portanto, reduz a exposição de carcinógenos fecais na superfície mucosa do cólon ${ }^{13}$. Outro mecanismo de prevenção pode estar relacionado com os níveis de prostaglandinas e de ácidos biliares fatores envolvidos na proliferação e crescimento de células do cólon -, sendo sugerido que a AF pode regular os níveis desses fatores de maneira benéfica ${ }^{37,38}$.

\section{CONCLUSÃO}

O presente estudo observou que, após as análises brutas e ajustadas para os fatores de confusão, a AF em seus quatro domínios (lazer, doméstica, ocupacional e deslocamento) e a AF total, realizadas durante toda a vida, não estiveram associadas ao desfecho. Os resultados analisados neste estudo foram contrários, em sua maior parte, ao encontrado na literatura; as possíveis explicações foram o perfil de AF na população estudada, as características da AF ocupacional e o tamanho amostral.

\section{CONTRIBUIÇÕES}

Todos os autores contribuíram substancialmente na concepção e/ou planejamento do estudo; na obtenção, análise e/ou interpretaçáo dos dados; na redação e revisão crítica; e aprovaram a versão final a ser publicada.

\section{DECLARAÇÃO DE CONFLITO DE INTERESSES}

Nada a declarar.

\section{FONTES DE FINANCIAMENTO}

Não há.

\section{REFERÊNCIAS}

1. Habr-Gama A. Câncer coloretal: a importância de sua prevenção. Arq Gastroenterol. 42(1):2-3. doi: https://doi. org/10.1590/S0004-28032005000100002

2. Instituto Nacional de Câncer José Alencar Gomes da Silva. Estimativa 2020: incidência de câncer no Brasil. Rio de Janeiro: INCA; 2019
3. Instituto Nacional de Câncer. Câncer no Brasil: dados dos registros de base populacional [Internet]. Rio de Janeiro: INCA; 2010 [acesso $2021 \mathrm{dez}$ 15]. Vol. 4. Disponível em: https://www.inca.gov.br/sites/ufu.sti.inca.local/ files//media/document//registro_de_base_populacional_ completo.pdf

4. Hoffman-Goetz L, Arumugam Y, Sweeny L. Lymphokine activated killer cell activity following voluntary physical activity in mice. J Sports Med Phys Fitness. 1994;34(1):83-90.

5. Boyle P, Leon ME. Epidemiology of colorectal cancer. Br Med Bull. 2002;64(1):1-25. doi: https://doi. org/10.1093/bmb/64.1.1

6. Sandler RS. Epidemiology and risk factors for colorectal cancer. Gastroenterol Clin North Am. 1996;25(4):717-35. doi: https://doi.org/10.1016/s0889-8553(05)70271-5

7. Diretrizes da OMS para atividade física e comportamento sedentário: num piscar de olhos [WHO guidelines on physical activity and sedentary behavior: at a glance] [Internet]. Geneva: World Health Organization; 2020. [cited 2021 Mar 15]. 24 p. Available from: https://apps.who.int/iris/ bitstream/handle/10665/337001/9789240014886-por. pdf? sequence $=102 \&$ isAllowed $=y$ Portugues .

8. Ministério da Saúde (BR). Vigitel Brasil 2019: vigilância de fatores de risco e proteção para doenças crônicas por inquérito telefônico: estimativas sobre frequência e distribuição sociodemográfica de fatores de risco e proteção para doenças crônicas nas capitais dos 26 estados brasileiros e no Distrito Federal em 2019 [Internet]. Brasília, DF: Ministério da Saúde; 2020 [acesso 2021 mar 15]. Disponível em: https://portalarquivos.saude. gov.br/images/pdf/2020/April/27/vigitel-brasil-2019vigilanciafatores-risco.pdf

9. Cummings JH, Bingham SA. Diet and the prevention of cancer. BMJ. 1998;317(7173):1636-40. doi: https:// doi.org/10.1136/bmj.317.7173.1636

10. Albanes D, Blair A, Taylor PR. Physical activity and risk of cancer in the NHANES I population. Am J Public Health. 1989;79(6):744-50. doi: https://doi. org/10.2105/ajph.79.6.744

11. Grant WB. Comments on E. Giovannucci, "Insulin, insulin-like growth factors and colon cancer: a review of the evidence”. J Nutr. 2002;132(8):2324. doi: https:// doi.org/10.1093/jn/132.8.2324

12. Giovannucci E. Insulin, insulin-like growth factors and colon cancer: a review of the evidence. J Nutr. 2001;131(11 Suppl):3109S-20S. doi: https://doi. org/10.1093/jn/131.11.3109S

13. Friedenreich CM, Orenstein MR. Physical activity and cancer prevention: etiologic evidence and biological mechanisms. J Nutr. 2002;132(11 Supl):3456S-64S. doi: https://doi.org/10.1093/jn/132.11.3456S 
14. Wolin KY, Yan Y, Colditz GA, et al. Physical activity and colon cancer prevention: a meta-analysis. Br J Cancer. 2009;100(4):611-6. doi: https://doi.org/10.1038/ sj.bjc. 6604917

15. Schmid D, Leitzmann MF. Television viewing and time spent sedentary in relation to cancer risk: a meta-analysis. J Natl Cancer Inst. 2014;106(7):dju098. doi: https://doi. org/10.1093/jnci/dju098

16. Baena R, Salinas P. Diet and colorectal cancer. Maturitas. 2015;80(3):258-64. doi: https://doi.org/10.1016/j. maturitas.2014.12.017

17. Robsahm TE, Falk RS, Heir T, et al. Cardiorespiratory fitness and risk of site-specific cancers: a long-term prospective cohort study. Cancer Med. 2017;6(4):86573. doi: https://doi.org/10.1002/cam4.1043

18. Friedenreich CM, Courneya KS, Bryant HE. The lifetime total physical activity questionnaire: development and reliability. Med Sci Sports Exerc. 1998;30(2):266-74. doi: https://doi.org/10.1097/00005768-199802000-00015

19. Ainsworth BE, Haskell WL, Herrmann SD, et al. 2011 Compendium of physical activities: a second update of codes and MET values. Med Sci Sports Exerc. 2011;43(8):1575-1581.

20. Hastert TA, White E. Association between meeting the WCRF/AICR cancer prevention recommendations and colorectal cancer incidence: results from the vital cohort. Cancer Causes Control. 2016;27(11):1347-59. doi: https://doi.org/10.1007/s10552-016-0814-6

21. Wei EK, Colditz GA, Giovannucci EL, et al. Cumulative risk of colon cancer up to age 70 years by risk factor status using data from the nurses' health study. Am J Epidemiol. 2009;170(7):863-72. doi: https://doi.org/10.1093/aje/ kwp210

22. Jurado D, Bravo LM, Cerón C, et al. Hábitos de vida y cáncer colorrectal: un estudio de casos y controles en una población de ingresos medios y bajos. Univ Salud. 2015;17(1):7-17.

23. Mafiana RN, Al Lawati AS, Waly MI, et al. Association between dietary and lifestyle indices and colorectal cancer in Oman: a case-control study. Asian Pac J Cancer Prev. 2018;19(11):3117-22. doi: https://doi.org/10.31557/ APJCP.2018.19.11.3117

24. Friedenreich C, Norat T, Steindorf K, et al. Physical activity and risk of colon and rectal cancers: the european prospective investigation into cancer and nutrition. Cancer Epidemiol Biomarkers Prev. 2006;15(12):2398-407. doi: https://doi.org/10.1158/1055-9965.EPI-06-0595

25. Leenders M, Siersema PD, Overvad K, et al. Subtypes of fruit and vegetables, variety in consumption and risk of colon and rectal cancer in the European Prospective Investigation into Cancer and Nutrition. Int J Cancer. 2015;137(11):2705-14. doi: https://doi.org/10.1002/ ijc. 29640
26. Wu S, Feng B, Li K, et al. Fish consumption and colorectal cancer risk in humans: a systematic review and meta-analysis. Am J Med. 2012;125(6):551-9.e5. doi: https://doi.org/10.1016/j.amjmed.2012.01.022

27. Sieri S, Krogh V, Agnoli C, et al. Dietary glycemic index and glycemic load and risk of colorectal cancer: results from the EPIC-Italy study. Int J Cancer. 2015;136(12):2923-31. doi: https://doi.org/10.1002/ ijc. 29341

28. Vulcan A, Manjer J, Ohlsson B. High blood glucose levels are associated with higher risk of colon cancer in men: a cohort study. BMC Cancer. 2017;17(1):842. doi: https:// doi.org/10.1186/s12885-017-3874-4

29. Liu L, Shi Y, Li T, et al. Leisure time physical activity and cancer risk: evaluation of the WHO's recommendation based on 126 high-quality epidemiological studies. Br J Sports Med. 2016;50(6):372-8. doi: https://doi. org/10.1136/bjsports-2015-094728

30. Harriss DJ, Atkinson G, Batterham A, et al. Lifestyle factors and colorectal cancer risk (2): a systematic review and meta-analysis of associations with leisure-time physical activity. Colorectal Dis. 2009;11(7):689-701. doi: https://doi.org/10.1111/j.1463-1318.2009.01767.x

31. Mahmood S, English DR, MacInnis RJ, et al. Domainspecific physical activity and the risk of colorectal cancer: results from the Melbourne Collaborative Cohort Study. BMC Cancer. 2018;18:1063. doi: https://doi. org/10.1186/s12885-018-4961-x

32. Martínez ME, Giovannucci E, Spiegelman D, et al. Leisure-time physical activity, body size, and colon cancer in women. Nurses' Health Study Research Group. J Natl Cancer Inst. 1997;89(13):948-55. doi: https://doi. org/10.1093/jnci/89.13.948

33. Keum N, Bao Y, Smith-Warner SA, et al. Association of physical activity by type and intensity with digestive system cancer risk. JAMA Oncol. 2016;2(9):1146-53. doi: https://doi.org/10.1001/jamaoncol.2016.0740

34. Rangul V, Sund ER, Mork PJ, et al. The associations of sitting time and physical activity on total and site-specific cancer incidence: results from the Hunt Study, Norway. PloS One. 2018;13(10):e0206015. doi: https://doi. org/10.1371/journal.pone.0206015

35. McTiernan A, Ulrich C, Slate S, et al. Physical activity and cancer etiology: associations and mechanisms. Cancer Causes Control. 1998;9(5):487-509. doi: https:// doi.org/10.1023/a:1008853601471

36. Oyeyemi SO, Braaten T, Licaj I, et al. Physical activity patterns and the risk of colorectal cancer in the Norwegian Women and Cancer study: a populationbased prospective study. BMC Cancer. 2018;18(1):1216. doi: https://doi.org/10.1186/s12885-018-5092-0

37. Ochsenkuhn T, Bayerdörffer E, Meining A, et al. Colonic mucosal proliferation is related to serum 
deoxycholic acid levels. Cancer. 1999;85(8):16649. doi: https://doi.org/10.1002/(SICI)10970142(19990415)85:8<1664::AID-CNCR4>3.0.CO;2-O

38. Wertheim BC, Martínez ME, Ashbeck EL, et al. Physical activity as a determinant of fecal bile acid levels. Cancer Epidemiol Biomarkers Prev. 2009;18(5):1591-8. doi: https://doi.org/10.1158/1055-9965.EPI-08-1187

Recebido em 3/2/2021

Aprovado em 17/3/2021 